\title{
Patterned running as a function of the sequence of trial administration '
}

JAMES H. McHOSE

SOUTHERN ILLINOIS UNIVERSITY

Groups of $S$ and $T$ received 201 trials on a recursive $R N N$ schedule of reinforcement. Group $T$, in which each $S$ received its first daily trial before the first $S$ received its second trial, etc., ran fast on $R$ and slow on $N$ trials. Group $S$, in which each $S$ received all of its daily trials, before the second $S$ received its first trial, ran fast, slow, fast on $R, N_{1}$, and $N_{2}$ trials, respectively.

Previous runway studies have shown that rats develop patterned running behavior under single alternation (SA) schedules of reward (R) and nonreward (N), running faster on $R$ than on $N$ trials (cf., Capaldi \& Stanley, 1963). SA patterning has been attributed to the development of a discrimination based upon either internal (cf., Capaldi \& Stanley) or external (Surridge \& Amsel, 1965) cues which are present at the outset of any trial and which are differentially associated with reinforcement. The internal cues differentially reinforced, according to Capaldi, are differential stimulus aftereffects from an $R\left(S_{R}\right)$ or an $N\left(S_{N}\right)$ on the preceding trial. One descriptive advantage of Capaldi's internal cue hypothesis derives from the fact that whether $S_{R}$ and $S_{N}$ are differentially associated with reinforcement depends upon the (trial to trial) reinforcement cycle, while the external cues specified by Surridge and Amsel (differential experimenter-produced cues preceding $\mathbf{R}$ as opposed to $\mathrm{N}$ trials or food odor cues) would be differentially reinforced regardless of the reinforcement schedule. The stimulus aftereffects hypothesis is thus peculiarly capable of accounting for the absence of patterning with double alternation reinforcement schedules as well as the observation that rats run slow, fast, fast, respectively, on a recursive NNR reinforcement schedule (Capaldi \& Senko, 1962).

The present study was concerned with an external cue interpretation of patterning behavior in which it is assumed that Ss exude differential odors on $\mathrm{N}$ as opposed to $R$ trials, and that these odors persist until the next $\mathrm{R}$ or $\mathrm{N}$ administration and thus, depending on the reinforcement cycle and the order in which Ss receive trials, serving as cues for reward or nonreward (McHose \& Ludvigson, 1966).

A unique implication of the olfactory hypothesis is that the specific patterning behavior resulting from any reinforcement schedule will depend upon the order in which Ss within a running group (or squad) of Ss receive their daily trials. The present study manipulated the trial order variable between groups which received a recursive RNN schedule of reinforcement.

\section{Method}

Fourteen male albino rats received 201 trials in a 55 in. long straight runway comprised of a gray start box (SB), and black alley and goal box (GB) sections (cf., McHose \& Ludvigson, 1966, for details). Opening of the door separating the alley section initiated a clock which stopped when $S$ interrupted a photocell beam located 6 in. into the alley section, providing a start time measure. Clock and photoelectric circuitry also provided traversal times over the next $12 \mathrm{in.} \mathrm{(running}$ time) and last $15 \mathrm{in}$. of the apparatus (goal time).

All Ss were placed on a $23 \mathrm{~h}$ food deprivation cycle 12 days prior to the first experimental day (Day 13). On Days 11 and 12, approximately $1 \mathrm{~g}$ of $45 \mathrm{mg}$ Noyes pellets, identical with the subsequent reinforcement pellet, was incorporated into Ss' daily diet, and Ss were allowed to explore the SB and alley sections of the apparatus for $3 \mathrm{~min}$.

All Ss received three trials per day for the first three days and six trials per day thereafter on a recursive RNN schedule of reinforcement. The first trial of any day was always an $R$ trial for all Ss. In Group $S$ $(N=6)$ each $S$ received all of its daily trials before the second $S$ in this group received its first daily trial, etc. In Group $T(N=8)$ each $S$ received the first daily trial before the first $S$ in this group recelved its second trial, etc. Reinforcement consisted of $20 \mathrm{sec}$ access to Noyes pellets. Ss were confined to GB for $20 \mathrm{sec}$ on $\mathrm{N}$ trials. Ss in Group $S$ were placed on a waiting stand adjacent to SB for $30 \mathrm{sec}$ between trials. The intertrial interval for Ss in Group T was approximately 350 sec.

Individual start, run, and goal times were reciprocated, yielding three speed measures on each trial. In addition, individual combined latencies on each trial were reciprocated to total speed scores.

Results

Group mean start, run, goal, and total speeds over Trials 142-201 for Groups $S$ and $T$ are presented in

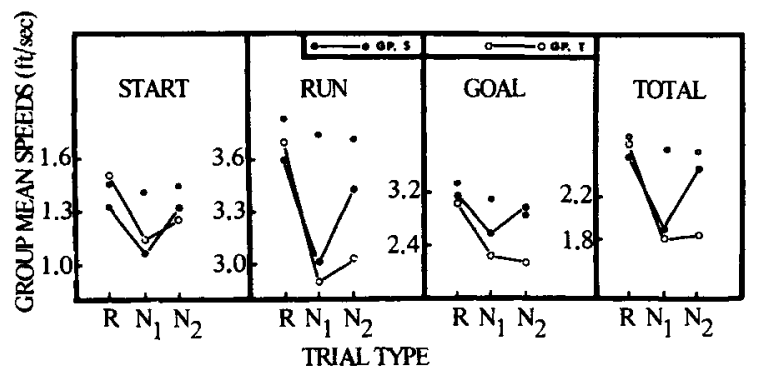

Fig. 1. Speeds in the different alley segments as a function of trial type. 
Fig. 1. For each group, speeds on the $R$, first $N\left(N_{1}\right)$, and second $\mathrm{N}\left(\mathrm{N}_{2}\right)$ trials of the $\mathrm{RNN}$ schedule are plotted separately. As may be seen, Group $S$ speeds were relatively fast on both $R$ and $N_{2}$ trials and slow on $N_{1}$ trials in all measures, while Group $\mathrm{T}$ speeds were relatively fast on $R$, but slow on $\mathrm{N}_{1}$ and $\mathrm{N}_{2}$ trials in all but the start measure. Variance analyses yielded a significant $(\alpha=.05)$ Trial Type by Groups interaction in the goal and total measures ( $F=7.43$ and 5.42 , respectively), and an interaction term approaching significance in the run measure $(F=2.58, d f=2 / 22)$. Analysis of start speeds yielded only a significant Trial Type effect ( $F$ $=11.73$, $d f=2 / 22)$. Further analyses of run, goal, and total measures within the two groups yielded a significant Trial Type effect in each measure for Group $T$ $(F=8.54,28.78$, and 14.50 , df $=2 / 12$; for Group $S, F=$ $8.10,9.12$, and 14.46, for run, goal, and total measures respectively). With respect to the run measure, paired comparison ( $t$ ) tests indicated that mean speeds on $\mathrm{N}_{1}$ and $\mathrm{N}_{2}$ trials did not significantly differ for Group $T$, nor did speeds on $R$ and $N_{2}$ trials differ for Group $S$.

In the above analyses the data for the first $S$ in Group $T$, the means of which appear as unconnected points in Fig. 1, were excluded for reasons to be discussed later. Discussion

The present results suggest that the patterning behavior developed in later segments of the alley depends upon the manner in which Ss receive trials. Animals which received all of their daily trials in succession, uninterrupted by trials for other Ss, ran fast, slow, fast on $R, N_{1}$, and $N_{2}$ trials, respectively. However, Group $T$, in which any trial for each $S$ was preceded by a trial on which a different $S$ received the same $R$ or $N$ event, ran fast, slow, slow on $R, N_{1}$, and $N_{2}$ trials. The behavioral patterning of Group $S$ is analogous to that obtained by Capaldi \& Senko (1962) on a recursive NNR cycle in which Ss received trials in the same fashion as did those of the present Group S. It should be noted that the total speed measure in the present experiment most nearly corresponds to the measure reported by Capaldi and Senko.

The behavioral patterning of Groups $S$ and $T$ is consistent with the assumption that Ss exude different odors on $\mathrm{R}$ and $\mathrm{N}$ trials and that these odors persist until the next $\mathrm{R}$ or $\mathrm{N}$ event is administered, whether to the same or a different S. For Ss in Group S, locomotion in the presence of perseverative $R$ odors are never reinforced, while locomotion in the presence of $N$ odors are partially (50\%) reinforced. As a result of differential reinforcement, then, $\mathrm{Ss}$ should run fast on $\mathrm{R}$ and $\mathrm{N}_{2}$ trials ( $\mathrm{N}$ odor trials) and slow on $\mathrm{N}_{1}$ trials (R odor trials). For $\mathrm{Ss}$ in Group $T$, however, perseverative R odors (exuded by the preceding $S$ ) and perseverative $\mathrm{N}$ odors are regularly associated with $R$ and $N$, respectively. As a result of differential reinforcement of these odor cues, Ss in Group $\mathrm{T}$ should run fast on $\mathrm{R}$ trials, but slow on $\mathrm{N}_{1}$ and $\mathrm{N}_{2}$ trials. An exception to this prediction would be expected with the first $S$ in a running group or squad of Ss since this $S$ has an odor-reinforcement contingency like that of Group S. Data relevant to this expectation were available from only one $S$ in the present study. That $S$, the first in Group $T$, displayed a pattern unlike that of either Groups $T$ or $S$.

While the patterning early in the instrumental response chain dictates an internal cue interpretation, responding in later segments of the chain was apparently cued, for any one $S$, by external stimuli produced on the preceding trial, whether administered to that Sor a different $S$. The fact that the relative import of these external cues was greater near GB is consistent with the notion that these cues are perseverative odors exuded by Ss on preceding trials in response to $R$ or $N$. The present data do not rule out the possibility that internal cues may suffice in patterned learning. They do clearly indicate, however, that any unequivocal demonstration of patterning behavior dependent only upon internal cues must control for "odor trail." The latter observation would seem particularly relevant to the question of whether (internal) stimulus traces persist over $24 \mathrm{~h}$ intertrial intervals (cf., Surridge \& Amsel, 1965).

Finally, in the present study the interval between trial administrations, whether between or within Ss, was controlled between groups, thus creating a confounding of intertrial interval with the trial order variable. The possibility that the difference between patterns developed by Groups $S$ and $T$ could be attributed to an ITI difference seems remote in view of a study reported by Ludvigson \& Sytsma (1967) in which manipulation of trial order, with ITI controlled, produced analogous differential behavior patterning on a double alternation schedule of reinforcement.

\section{References}

CAPALDI, E. J., \& SENKO, M. G. Acquisition and transfer in partial reinforcement. J. exp. Psychol, 1962, 63, 155-159.

CAPALDI, E. J., \& STANLEY, L. R. Temporal properties of reinforcement aftereffects. $J$. exp. Psychol, 1963, 65, 169-175.

LUDVIGSON, H. W., \& SYTSMA, D. The sweet smell of success: Apparent double alternation in the rat. Paper presented at the annual convention of the Midwest. Psychol. Assoc., Chicago, 1967.

McHOSE, J. H., \& LUDVIGSON, H. W. Differential conditioning with nondifferential reinforcement. Psychon Sci, 1966, 6, 485-486.

SURRIDGE, C. T., \& AMSEL, A. Performance under a single alternation schedule of reinforcement at 24hour intertrial intervals. Psychon. Sci, 1965, 3, 131-132.

Note

1. Supported by grant MH 10340 from the United States Public Health Service. 